\title{
Observation et modélisation de la houle sur la plage macrotidale de Pentrez en baie de Douarnenez.
}

\author{
David Morellato $^{1,2}$, Georges Chapalain ${ }^{2}$ et Nicolas Guillou ${ }^{1,2}$ \\ ${ }^{1}$ CETMEF - Technopôle Brest-Iroise - BP 5 - 29280 Plouzané, \\ France David.Morellato@equipement.gouv.fr, \\ Nicolas.guillou@equipement.gouv.fr \\ ${ }^{2}$ UMR CNRS-UBO 6539 LEMAR - Institut Universitaire Européen \\ de la Mer - Place Nicolas Copernic-29280 Plouzané, France \\ David.Morellato@univ-brest.fr, Georges.Chapalain@univ-brest.fr, \\ Nicolas.Guillou@univ-brest.fr
}

\section{Résumé}

Lors de la campagne EPIES (avril 2005), des mesures ont été réalisées aux abords et sur la plage sableuse macrotidale de Pentrez, en baie de Douarnenez, à l'aide d'une bouée-houlographique mouillée par $12 \mathrm{~m}$ de fond et d'un courantomètre électromagnétique doublé d'un capteur de pression (S4DW) déployé dans la partie basse de la zone intertidale $(z=+3 \mathrm{~m})$. Ces mesures sont analysées et exploitées dans une démarche de modélisation numérique fondée sur les équations du modèle de Boussinesq étendu (Wei et al., 1995 $5^{10}$ ) (modèle FUNWAVE, Kirby et al., $\left.1998^{6}\right)$. Cette approche de modélisation est confrontée avec un modèle spectral de propagation et discutée en termes de prévision des caractéristiques de houle à l'approche de la côte.

\begin{abstract}
During the EPIES campaign (April, 2005), measurements were collected off the macrotidal sandy beach of Pentrez, in the bay of Douarnenez, with the help of a waverider buoy (depth of $-12 \mathrm{~m}$ ) and an electromagnetic current-meter plus a pressure sensor moored in the shoreface (S4DW) deployed in the low part of the intertidal zone $(\mathrm{z}=+3 \mathrm{~m})$. These measures are analyzed and exploited in a numerical modelling approach based on the extended Boussinesq equations (Wei et al., 1995 ${ }^{10}$ ) (model FUNWAVE, Kirby et al., 19986). This approach is confronted with a spectral propagation model and discussed in terms of prediction of the parameters of waves approaching the shoreline.
\end{abstract}

Mots clés : houle, plage macrotidale, Equations de Boussinesq, modèle numérique, mesure.

\section{Introduction}

En ingénierie côtière, il est important de connaître les paramètres décrivant l'état de mer à la côte en vue d'appréhender l'impact de la houle et par suite d'élaborer 
des stratégies d'aménagement et de défense. Ces paramètres sont évalués, soit par mesure directe à l'aide de bouées houlographiques, soit à l'aide de modèles de propagation. Les modèles récents les plus communément utilisés sont basés sur l'équation d'évolution de la densité spectrale d'action (Hasselmann et al., 1973 ${ }^{4}$ ). Parmi ceux-ci, citons le modèle SWAN (Booij et al., 2004 ${ }^{\mathbf{1}}$ ). Des paramètrisations sont introduites pour incorporer les effets liés au "shoaling ", aux non-linéarités, au frottement sur le fond et au déferlement. Néanmoins, ces modèles énergétiques traitant des variables moyennées s'avèrent limités pour appréhender de manière détaillée les processus hydrodynamiques et hydro-sédimentaires associés à la houle en eau peu profonde. Une alternative est offerte par la résolution des équations de Boussinesq $\left(1872^{2}\right)$. En vue d'analyser l'impact de la houle sur la dynamique littorale, un code de Boussinesq a été implémenté et étendu au calcul des processus hydro-sédimentaires, puis de l'évolution morphologique de l'avantplage et de la plage via la résolution de l'équation de conservation du sédiment. La présente note est consacrée à la première étape de ce travail qui consiste à mettre en œuvre le code de Boussinesq et à confronter ses résultats avec les mesures recueillies en baie de Douarnenez ainsi qu'avec les résultats issus du code spectral SWAN.

\section{Observations}

\section{1. Site d'étude}

La plage de Pentrez, au fond de la baie de Douarnenez (Figure 1), est le site d'étude retenu. Cette plage de sable fin $\left(\mathrm{d}_{50}=140 \mu \mathrm{m}\right)$ est soumise à de faibles courants de marée ( inférieurs à $5 \mathrm{~cm} / \mathrm{s}$ en condition de vive-eau) et à de fortes houles océaniques qui constituent l'agent hydrodynamique prédominant. Elle présente une pente constante de 1 à $2 \%$ et peu de variabilité longitudinale sur ses deux kilomètres de linéaire. Il s'agit enfin d'une plage de type « dissipatif» avec des déferlements de type « glissant».

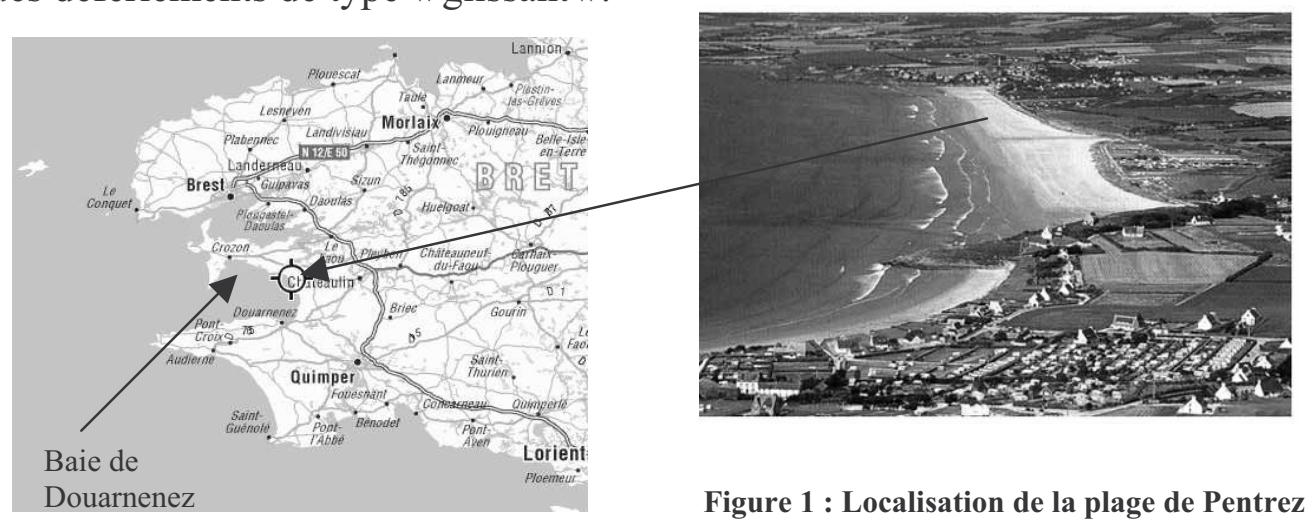

\section{2. Mesures effectuées}

Dans le cadre de la campagne EPIES consacrée à la mesure des flux sédimentaires en zone subtidale, trois houlographes et une série de courantomètres 
électromagnétiques et acoustiques ont été déployés du 15 au 24 avril 2005 en mer d'Iroise et en baie de Douarnenez. Une des bouées (DW3) a été mouillée par $12 \mathrm{~m}$ de fond à $2300 \mathrm{~m}$ de la ligne de rivage. Du 19 au 21 avril, en conditions de revif de marée (coefficients passant de 42 à 62), et de tempête molissante, le dispositif expérimental a été complété par un courantomètre-houlographe S4DW installé en zone intertidale sur la plage de Pentrez à $30 \mathrm{~cm}$ au-dessus du fond. La bouée DW3 acquiert en mode salve («burst»), à la cadence de $2 \mathrm{~Hz}$ pendant 20 minutes, toutes les 30 minutes, tandis que le S4DW mesure en continu à la cadence de $2 \mathrm{~Hz}$. En parallèle aux mesures in-situ, un suivi topographique de la plage a été entrepris en collaboration avec le laboratoire GEOMER. Du Modèle Numérique de Terrain (MNT) levé le 27 avril 2005, complété en zone subtidale par la bathymétrie du SHOM, nous extrayons le profil de plage instrumenté présenté à la Figure 2. La hauteur d'eau moyennée sur 10 minutes, la hauteur significative spectrale et la période de pic d'énergie de la houle sont montrées à la Figure 3. On constate la décroissance des conditions de houle au cours du temps.

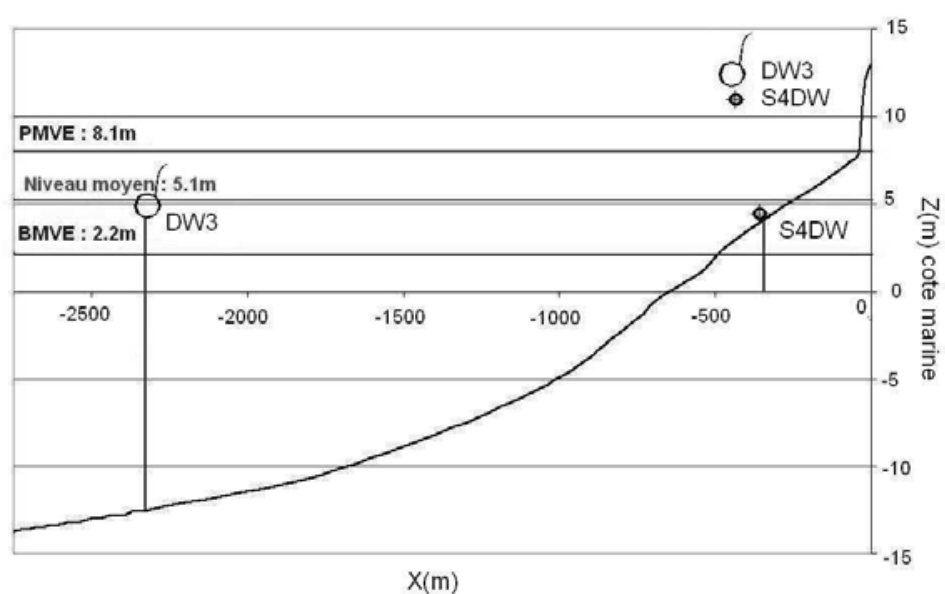

Figure 2 : Localisation des instruments
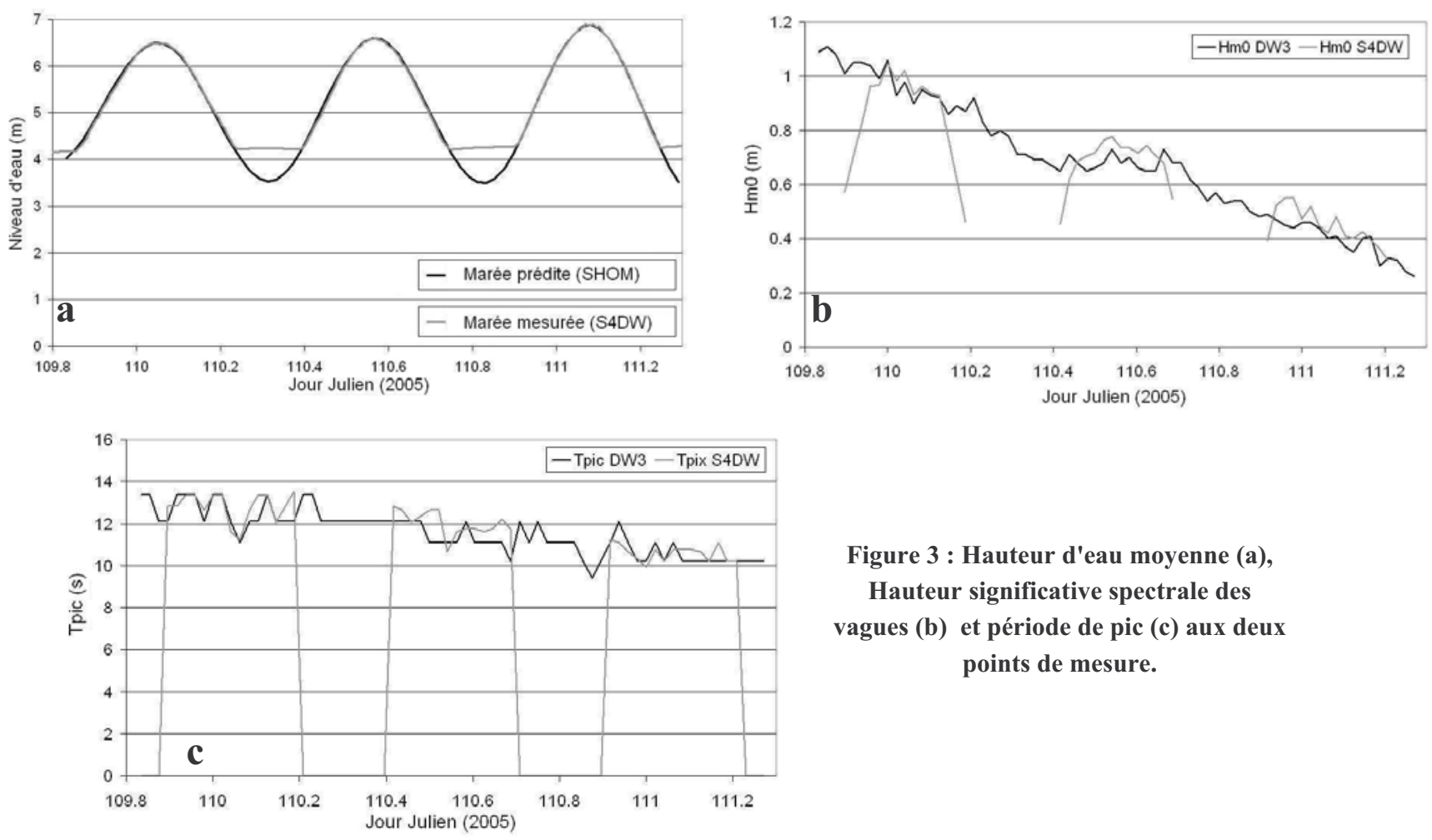

Figure 3 : Hauteur d'eau moyenne (a),

Hauteur significative spectrale des

vagues (b) et période de pic (c) aux deux points de mesure. 


\section{Modélisation}

La modélisation consiste dans un premier temps à appliquer un modèle de Boussinesq unidimensionnel (1D) le long du profil de plage instrumenté, de la position de la bouée DW3, jusqu'au rivage; la chronique du niveau d'eau enregistrée par la bouée DW3 étant « injectée » comme condition à la limite extérieure du domaine de calcul. Les résultats du modèle sont ensuite comparés aux mesures effectuées sur la plage par le S4DW. Dans un second temps, un modèle spectral est appliqué sur le même domaine, puis sur un domaine étendu à la baie de Douarnenez.

\section{1. Modèle de Boussinesq}

Le modèle de Boussinesq retenu est le modèle FUNWAVE développé à 1'université du Delaware (Kirby et al., 19986 ${ }^{6}$ ). C'est un modèle numérique aux différences finies qui décrit la surface libre et la vitesse du courant à une profondeur donnée, en absence de déferlement sur la base des équations de la houle longue de Wei et al. $\left(1995^{\mathbf{1 0}}\right)$ suivantes :

$$
\begin{aligned}
& \eta_{t}+\left\{(h+\eta) u_{\alpha}+\left(z \alpha+\frac{1}{2}(h-\eta)\right)\left(h u_{\alpha}\right)_{x x}+\left(\frac{1}{2} z_{\alpha}^{2}-\frac{1}{6}\left(h^{2}-h \eta+\eta^{2}\right)\right) u_{\alpha x x}\right\}_{x}=0 \\
& u_{\alpha t}+u_{\alpha} u \alpha x+g \eta_{x}+z \alpha\left\{\frac{1}{2} z \alpha u \alpha t x x+\left(h u_{\alpha t}\right)_{x x}\right\} \\
& +\left\{\frac{1}{2}\left(z_{\alpha}^{2}-\eta^{2}\right) u_{\alpha} u_{\alpha x x}+\frac{1}{2}\left[(h u \alpha)_{x}+\eta u_{\alpha x}\right]^{2}\right\}+\left\{(z \alpha-\eta) u_{x}(h u \alpha)_{x x}-\eta\left[\frac{1}{2} \eta u_{\alpha t x}+\left(h u_{\alpha t}\right)_{x}\right\}_{x}=0\right.
\end{aligned}
$$

où

$$
\begin{aligned}
& \text { x est l'abscisse ; } \\
& \text { t le temps ; } \\
& \eta \text { est la dénivellation de la surface libre; } \\
& \text { h est la profondeur d'eau; } \\
& \mathrm{z}_{\alpha}=0.531 \text { h est l'altitude où sont développées les équations ; } \\
& \mathrm{u}_{\alpha} \text { est le courant à l'altitude } \mathrm{z}_{\alpha} ; \\
& \text { g est l'accélération de la pesanteur. }
\end{aligned}
$$

La structure verticale du courant est donnée par :

$$
u(z)=u_{\alpha}+\frac{z_{\alpha}^{2}-z^{2}}{2}\left(u_{\alpha}\right)_{x x}+(z \alpha-z)(h u \alpha)_{x x}
$$

Au niveau du déferlement, l'excès d'eau apporté par la vague crée un courant au fond dirigé vers le large, non reproduit par le modèle. Supposant que le flux calculé par un modèle de Boussinesq est valide, Lynett $\left(2005^{7}\right)$ propose de corriger en post-traitement la structure verticale du courant (Eq. 2) par une fonction de type exponentiel et d'assimiler la vitesse en surface à celle du rouleau. 
Des paramétrisations ont été introduites pour incorporer plus complètement l'impact du déferlement (Kennedy et al. $\left(2000^{5}\right)$ et Chen et al. $\left(2000^{3}\right)$ ). Dans la zone de déferlement localisée grâce au concept du «Surface Roller », des termes de viscosité et de frottement en surface ont ainsi été ajoutés dans l'équation de conservation de quantité de mouvement (Eqs. 1b). Le « runup » des vagues sur la plage est par ailleurs pris en compte à l'aide d'une technique de plage perméable.

De la même façon, une contrainte de frottement sur le fond quadratique est incorporée dans le modèle :

$$
F_{b}=\frac{f_{w}}{h+\eta} u_{\alpha}\left|u_{\alpha}\right|
$$

dans laquelle $f_{\mathrm{w}}$ est le coefficient de frottement choisi égal à 0.002 , valeur proche de la gamme 0,003-0,004 obtenue en appliquant la formule de Soulsby(1997 $\left.{ }^{9}\right)$ $f_{w}=0.237\left(\frac{u(-h)^{*} T p i c}{2 \pi k_{s}}\right)^{-0.52}$ avec la hauteur de rugosité $\mathrm{k}_{\mathrm{s}}$ admise réduite à celle du grain $\left(2.5^{*} \mathrm{~d}_{50}\right)$ ignorant les rides sableuses conformément aux observations à marée basse sur la plage.

Un calcul est effectué pour chaque «burst» en supposant le niveau marin constant. Les pas spatial et temporel valent respectivement $1 \mathrm{~m}$ et $0.065 \mathrm{~s}$.

\section{2. Modèle spectral}

Le modèle de Boussinesq FUNWAVE est comparé au modèle de propagation spectral SWAN (Booij et al., 2004 $4^{\mathbf{1}}$ ). Deux simulations sont effectuées. La première simulation est réalisée en mode multi-stationnaire forcée par les données de la bouée DW3 sur la plage de Pentrez. Un calcul stationnaire a été répété toutes les 30 minutes avec un pas spatial de $10 \mathrm{~m}$. La seconde simulation est réalisée sur l'ensemble de la baie de Douarnenez en mode instationnaire, c'est à dire en termes de hauteur d'eau moyenne oscillant avec la marée et de houle incidente (mesurée à l'entrée de la baie). Le pas spatial est de $100 \mathrm{~m}$ et le pas temporel de 30 minutes. (cf Morellato et al., 2006 ${ }^{8}$ ).

\section{Résultats et discussions}

La Figure $3 a$ montre les hauteurs de houle significative spectrale $\mathrm{H}_{\mathrm{m} 0}$ issues des trois modélisations comparées aux mesures. Le meilleur accord est trouvé avec le modèle FUNWAVE. Les deux simulations issues du modèle SWAN surestiment les observations d'autant plus que le maillage est grand. La Figure $3 b$ présente les périodes de pic prédites et mesurées. Elle est généralement bien reproduite. La Figure 3c compare le courant à $30 \mathrm{~cm}$ au-dessus du fond calculé par FUNWAVE à celui mesuré par le S4DW. On remarque que la correction de Lynett $\left(2005^{7}\right)$ 
améliore la prédiction, sauf en eau très peu profonde. Lors du $3^{\text {ème }}$ cycle de marée, on constate que le courant est dirigé vers la plage lors du montant. Cela est sans doute imputable au courant de marée qui devient prépondérant devant les courants induits par la houle quand celle-ci est faible. La figure $3 \mathrm{~d}$ compare les écarts quadratiques sur la vitesse (vitesses RMS). Là aussi, cette variable est bien reproduite par les trois modélisations.
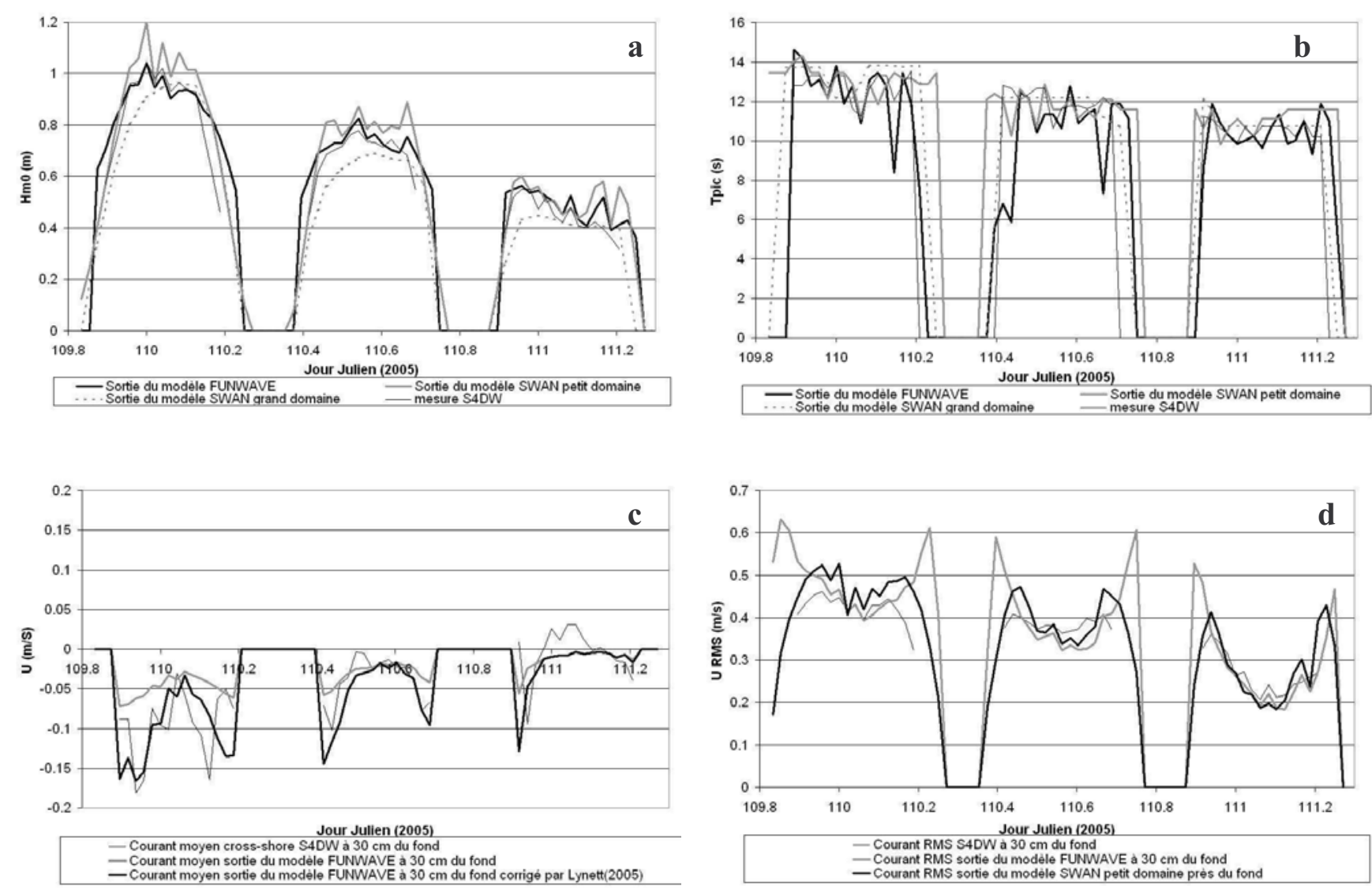

Figure 4 : Hauteur significative spectrale des vagues (a), période de pic (b), courant (c) et vitesse RMS (d) prédits et mesurés sur la plage de Pentrez

La Figure 5 présente un instantané de la houle le long du profil de plage : on remarque que la hauteur significative spectrale des vagues $H_{m 0}$ prédite par SWAN s'écarte de plus en plus de celle prédite par FUNWAVE au fur et à mesure que la profondeur diminue. 


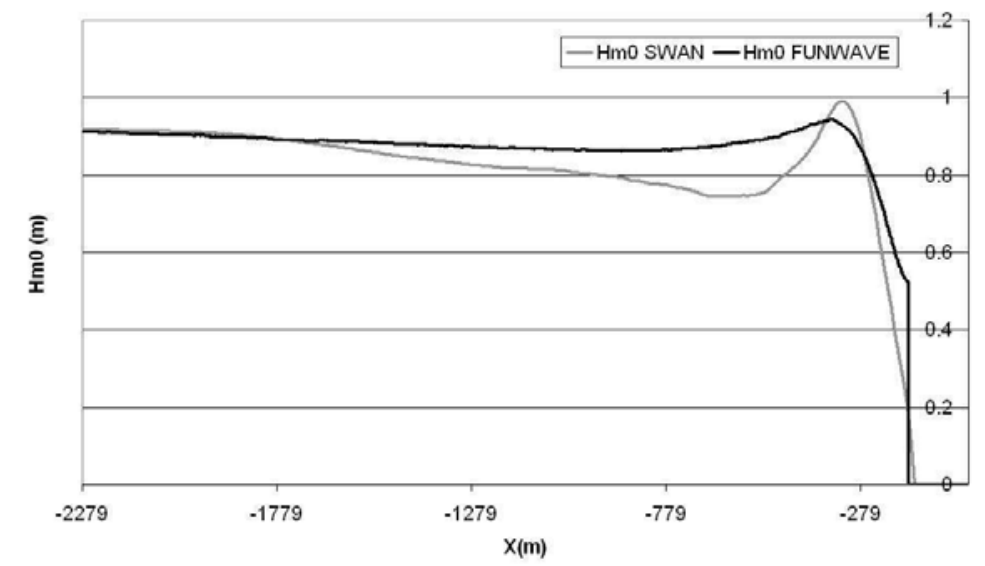

Figure 5 : Comportement de la houle le long du profil (20/04/2005 2H30 UTC)

\section{Conclusions}

La présente étude a montré les meilleures capacités prédictives du modèle vague par vague FUNWAVE par rapport au modèle spectral SWAN à prédire l'évolution des vagues dans les eaux peu profondes, tant en zone subtidale proche qu'en zone intertidale. Les mesures exploitées ne permettent pas toutefois de valider pleinement ce modèle. En effet, avec un volume de mesure d'environ $0,4 \mathrm{~m}$ de diamètre, les données de l'unique S4DW ne peuvent pas bien rendre compte de la variabilité spatiale, en particulier sur la verticale au niveau du courant de retour. De nouvelles mesures plus fines et plus nombreuses dans l'espace sont envisagées à court terme pour atteindre cet objectif et étendre la modélisation aux aspects hydro-sédimentaires, puis morphodynamiques.

\section{Remerciements}

Nous adressons nos vifs remerciements à Guy Amis (CETMEF) et Eric Duvieilbourg (UMR CNRS-UBO 6539 LEMAR), ainsi qu'à l'équipage du N/O "Côtes de la Manche" pour leur contribution à l'acquisition des mesures de terrain lors de la campagne EPIES. Nous remercions enfin Alain Henaff (UMR CNRS-UBO 6554 LETG-GEOMER) pour sa participation aux relevés topographiques sur la plage de Pentrez.

\section{Bibliographie}

1 Booij, N., Haagsma, IJ.G., Holthuijsen, L.H., Kieftenburg, A.T.M.M., Ris, R.C., Van der Westhuysen, A.J. and Zijlema, M. (2004). SWAN Cycle III version 40.41, USER MANUAL. Environmental Fluid Mechanics Section, 
Faculty of Civil Engineering and Geosciences, Delft University of Technology.

2 Boussinesq, J. (1872). Théorie des ondes et des remous qui se propagent le long d'un canal rectangulaire horizontal, J. Math. Pures. Appl., Vol.17, 55108.

3 Chen, Q., Kirby, J. T., Dalrymple, R. A., Kennedy, A. B. and Chawla, A. (2000). Boussinesq modeling of wave transformation, breaking and runup. II : Two horizontal dimensions. J. Waterway, Port, Coastal and Ocean Engrng., 126, 48-56.

4 Hasselmann K., Barnett, T. P., Bows, E., Carlson, H., Cartwright, E., Engke, K., Ewing, J. A., Gienapp, H., Hasselmann, D. E., Kruseman, P., Meerburg, A., Müller, P., Olbers, D. J., Richter, K., Sell, W. and Walden, H. (1973). Measurements of wind-wave growth and swell decay during the Joint North Sea Wave Project (JONSWAP). Deutsch Hydrographic Z. Suppl. 12, A8, 1-95.

5 Kennedy, A. B, Chen, Q., Kirby, J. T. and Dalrymple, R. A. (2000). Boussinesq Modelling of wave transformation, breaking and runup. I : One dimension. J. Waterway, Port, Coastal and Ocean Engrng., 126, 3947.

6 Kirby, J. T., Wei, G., Chen, Q., Kennedy, A. B. and Dalrymple, R. A. (1998). FUNWAVE 1.0. Fully nonlinear Boussinesq wave model. Documentation and user's manual, Report CACR-98-06. Center for Applied Coastal Research, Department of Civil and Environmental Engineering, University of Delaware.

7 Lynett, P. J. (2005). Wave Breaking velocity effects in depth-integrated models. Coastal Engineering, In Press.

8 Morellato, D., Guillou, N., Chapalain, G., Duvieilboug, E. et Amis, G. (2006, soumis) Intercomparaison et validation de différents modèles de propagation de houle en baie de Douarnenez. IXèmes Journées Nationales Génie Côtier-Génie Civil - Brest 2006

9 Soulsby, R.L. (1997). Dynamics of marine sands, a manual for pratical applications. Thomas Telford, H.R. Wallingford.

10 Wei, G. and Kirby, J. T. (1995). Time-dependent numerical code for extended Boussinesq equations. J. Waterway, Port, Coast, and Ocean Engng., 121(5), 251-261. 